\title{
Fabric Defect Detection by using Neural Network technique
}

\author{
${ }^{1}$ Prof. M. S. Biradar, ${ }^{2}$ Sharda P. Jadhav \\ ${ }^{1}$ HOD Department of Electronics, Siddhant College of Engg, Sudumbre, Pune \\ ${ }^{2}$ ME IInd Year Student, Siddhant College of Engg, Sudumbre, Pune
}

\begin{abstract}
This paper proposes a method for fabric defect detection based on neural network. The global market for textile industry is highly competitive nowadays. Neural network is widely used to extract features from images for texture segmentation. The proposed scheme involves two challenging problem i.e. defect detection and defect classification. Scene analysis and feature selection play an important role in classification process. The complexity of the subsequent steps increases and the classification task becomes hard by selecting an inappropriate feature set. So possibly an appropriate set of geometric features is taken into account in order to address the problem of neural network-based textile defect classification. Statistical approach is used to extract the features.
\end{abstract}

Key Words: Fabric Defect, Defect Detection, Neural network, Feature Extraction.

\section{Introduction}

Quality control is an important factor in industrial production which is increasing day by day. Textile industry is not an exception in this regard. Inspection of fabric defects plays an important role in the quality control. Human vision inspection is not enough accurate due to fatigue and tediousness. Also, it is time consuming and high quality cannot be maintained. So the solution to this problem is automated i.e. machine vision based textile inspection system. Automated textile inspection involves two challenging problem, namely, defect detection and defect classification. Classification process is composed of several steps. One of the important steps is scene analysis and feature selection. The complexity of the subsequent steps increases and the classification task becomes hard by selecting an inappropriate feature set.

Bangladesh, as a developing country specially focuses on export, through which a lot of foreign exchange is earned. Bangladesh textile industry is major export sector and strength of industry are cheap labor cost, low energy price, and good-quality products. In the highly competitive global market to increase the performance industry should increase productivity as well as quality. Quality is affected due to defects that occur in textile. So accurate detection of defect is an important factor to improve the quality.

Defect analysis is a very important part of our approach to automated textile defect inspection problem, which has been done earlier than all other parts. Defect analysis helps understand the defects properly, and give clues to appropriate feature. In this paper, we have dealt with four types of defects, which frequently occur in knitted fabrics in Bangladesh, namely, color yarn, hole, missing yarn, and spot.

Automated textile inspection systems are real-time applications. So they require real-time computation, which exceeds the capability of traditional computing. Neural networks (NNs) are suitable enough for real-time systems because of their parallel-processing capability. Moreover, NNs have strong capability to handle classification problems. The classification accuracy of an appropriate NN, which handles multiclass problems, is good enough. There is a number of performance metrics of NN models. Classification accuracy, model complexity and training time are three of the most important performance metrics of NN models.

Objective of this paper is to present an appropriate set of features in order to address the problem of defect classification. We justify the features in terms of their discriminatory qualities considering the difficulties which lie in the feature extraction process. We use statistical approach to extract the features.

\section{Neural Network}

A computing system made up of a number of simple, highly interconnected processing elements, which process information by their dynamic state response to external input.

Our basic computational element (model neuron)

Is often called a node or unit. It receives input from some other units, or perhaps from an external source. Each input has an associated weight $\mathrm{w}$, which can be modified so as to model synaptic learning. The unit computes some function $\mathrm{f}$ of the weighted sum of its inputs:

$$
y=\left(\begin{array}{ll}
w & y
\end{array}\right)
$$

Its output, in turn, can serve as input to other units 


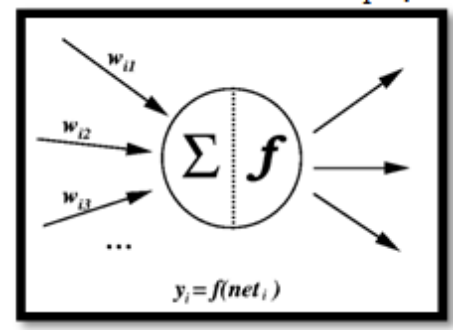

Figure.1.

To the second layer of neurons, and then via more synapses to the third layer of output neurons. More complex systems will have more layers of neurons with some having increased layers of input neurons and output neurons. The synapses store parameters called "weights" that manipulate the data in the calculations.

\section{Literature Review}

The reduction of wastage, higher price of fabrics due to the presence of fewer defects, requirement of less labor, and other benefits make the investment in an automated textile defect inspection system economically very attractive. The development of a fully automated web inspection system requires robust and efficient defect detection and classification algorithms. The inspection of real textile defects is particularly challenging due to the large number of textile defect classes, which are characterized by their vagueness and ambiguity. A number of attempts have been made for human-

The weighted sum $f\left(\begin{array}{ll}w & y\end{array}\right)$ is vision-based textile defect inspection. Most of them

called the net input to unit $i$, often written net.

Note that $w_{i j}$ refers to the weight from

unit $j$ to unit $i$ (not the other way around).

The function $f$ is the unit's activation function. In the simplest case, $f$ is the identity function, and the unit's output is just its net input. This is called a linear unit.

The function $f$ is the unit's activation function. In the simplest case, $f$ is the identity function, and the unit's output is just its net input. This is called a linear unit. For example, in a neural network for handwriting recognition, a set of input neurons may be activated by the pixels of an input image representing a letter or digit. The activations of these neurons are then passed on, weighted and transformed by some function determined by the network's designer, to other neurons, etc., until finally an output neuron is activated that determines which character was read.

Like other machine learning methods, neural networks have been used to solve a wide variety of tasks that are hard to solve using ordinary rule-based programming, including computer vision and recognition. The word network in the term 'artificial neural network' refers to the inter-connections between the neurons in the different layers of each system. An example system has three layers. The first layer has input neurons, which send data via synapses have concentrated on defect detection, where few of them have concentrated on classification. There are mainly three defect-detection techniques, namely, statistical, spectral, and model based [08].

A number of techniques have used for classification. Among them, neural network, support vector machine (SVM), clustering, and statistical inference [09] are notable. Scene analysis, that is, defect analysis, and feature Selection are basically relevant to the works, which have dealt with multiclass problem, that is, categorizing defects distinctly. 


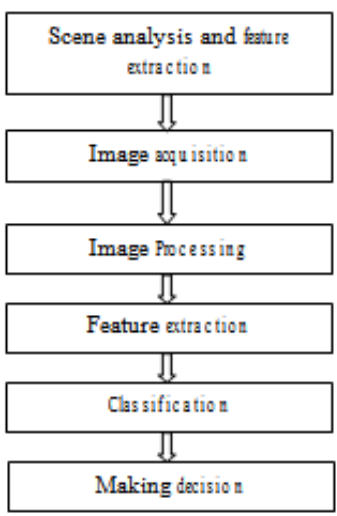

The development of automated system involves number of steps as shown in figure 2.Each step has effect on the performances of its preceding step. Each step has lot of importance in machine vision system. The task of Scene analysis and feature extraction is challenging issue. The complexity of the subsequent steps increases and the classification task becomes hard by selecting an inappropriate feature set. In the beginning of automated textile inspection system, various scene of different colored defective and defect free fabric should be analyzed. Then each defect occurred should be analyzed properly. This will facilitate selection of the features for classification. Each of the features should be properly justified in terms of their discriminatory qualities and complexities to extract them, which is also very challenging. This results in an appropriate feature set, which will make the system's performance good.

\section{Approach And Methodology Many Possible Approaches Are Investigated In Order}

[]o accomplish our task. Finally, we have found the approach, shown in Figure 2, optimal. Approach starts with an inspection image of knitted fabric which is of size $512 * 512$ pixel. Inspected image is converted into a grayscale image. In order to smooth the image and remove noises, image is filtered by $7 * 7$ low pass filter convolution mask. Then grayscale histogram of the image is formed and two threshold

values $\theta \mathrm{L}$ and $\theta \mathrm{H}$ are calculated from the histogram. Using this threshold values, image with pixel $\mathrm{p}(\mathrm{x}, \mathrm{y})$ is converted into a binary image with pixel $\mathrm{b}(\mathrm{x}, \mathrm{y})$ where whether the image is defect-free, or defective with the name of the defect. Then a number of features of the defect are calculated, which forms the feature vector corresponding to the defect in the image. Figure shows the stepwise changed images.

4.1. Defect analysis. In automated textile defect inspection problem Defect Analysis most important part. Through defect analysis we understand defects properly and gets clues to appropriate features. In this paper we referred to four types of defects which occur frequently in knitted fabric in Bangladesh, namely, colour yarn, hole, missing yarn, and spot. All of the defects are shown in fig.3.

i) Color yarn: Figure 3(a) shows the defect of color yarn. Color yarn is one of the smallest and sneakiest defects that occur in knitted fabrics in Bangladesh. It appears in a shape, close to a small rectangle of one color, on a fabric of another color. It becomes little blurred in its captured image.

(ii) Hole: Figure 3(b) shows the defect of hole. Hole is one of the most severe defects that occur in knitted fabrics in Bangladesh. It appears in a shape, close to a circle of the color of the background, on a fabric of another color. Its size varies from small to medium. The shape of the defect of hole can become a little distorted, for example, oval, if inappropriate viewpoint is chosen by positioning the camera improperly. The color of the background is another issue. In some cases, background color can become close to the color of fabric.

(iii) Missing yarn: Figure 3(c) shows the defect of

$\mathrm{b}(\mathrm{x}, \mathrm{y})=1, \quad$ ?의 $\theta$ ? $\leq \mathrm{p}(\mathrm{x}, \mathrm{y}) \leq \theta H$

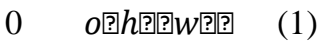

missing yarn. Missing yarn is also one of the most severe defects that occur in knitted fabrics in Bangladesh. It appears as a thin striped shade of the

This binary image contains defect (object) if any exists and defect free fabric (background), and some noises. These noises are removed using thresholding. In our approach, we intend to detect a defect of minimum size $3 \mathrm{~mm} \times 1 \mathrm{~mm}$. So, any object smaller than the minimum-defect size in pixels is eliminated from the binary image. If the minimum-defect size in pixels is $\theta \mathrm{MD}$ and an object with pixels $o(x, y)$ is of size $S o$ pixels, then

$\mathrm{O}(\mathrm{x}, \mathrm{y})=1, \quad 0 \geq$ 廆 $\quad D$ 
0, oh $w$

Then a feature vector is formed by calculating anumber of features of the defect. Then this feature vector is inputted to an artificial network which is trained earlier with a number of feature vectors, in order to classify the defect. Finally, it is out putted color of fabric. It is usually long. It is of two types, namely, vertical and horizontal. Proper lighting is required in order to clearly capture the image of the defect of missing yarn.

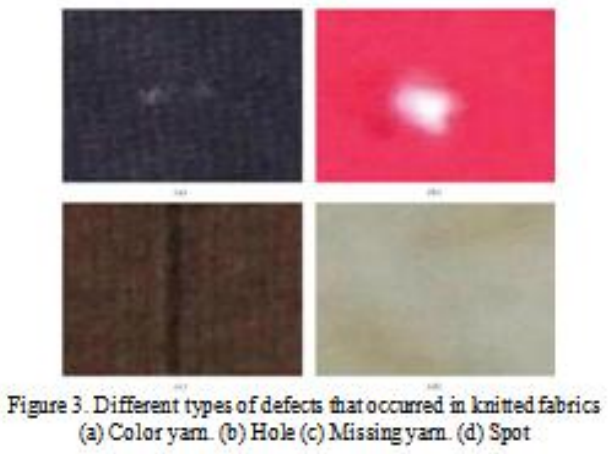

(iv) Spot: Figure 3(d) shows the defect of spot. Spot is one of the most eccentric defects that occur in knitted fabrics in Bangladesh because of its appearance and nature. It does not appear in any specific shape. It usually appears in a scattered form of one color on a fabric of another color. Moreover, its size varies widely, that is, from medium to large. It becomes little blurred in its captured image in some cases and does not become such blurred in other cases. A camera of high resolution and proper lighting is required in order to clearly capture the image.

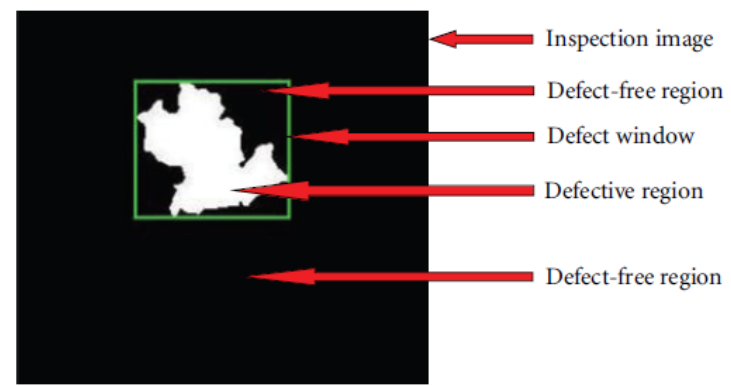

Figure 4: Terminology adopted

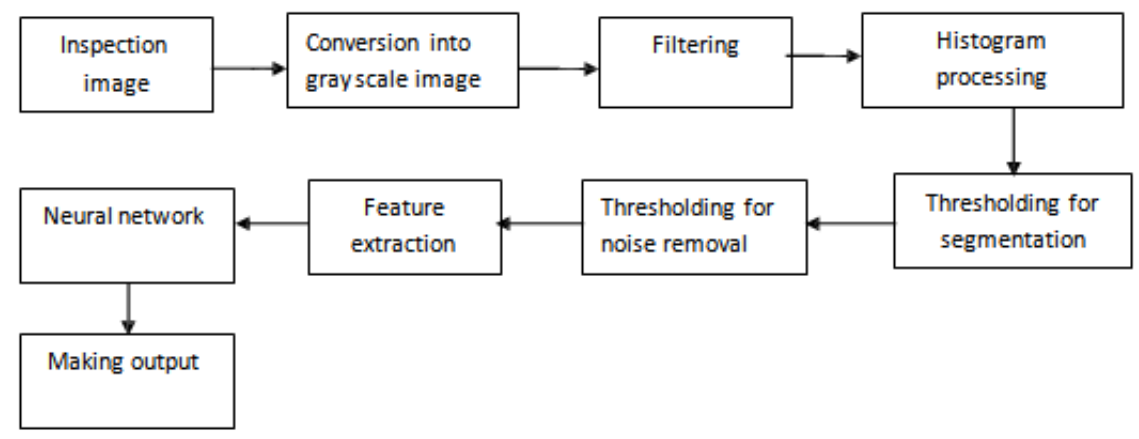

Figure 5: Block diagram of the textile defect inspection method

4.2. Terminology. Some terms are used for appropriate set of geometric features which are explained below and shown in figure $4 .:$

i) Inspection Image: Inspection image or image is the image to be inspected.

ii) Defective Region: Defective Region is the maximum connected area of defect in an inspection image.

iii) Defect-Free Region: Defect-free region is the maximum connected area in an inspection image, which does not contain any defect.

iv) Defect Window: Defect window is the rectangle of minimum area, which encloses all Defective Regions in an inspection image. Characteristics of the defect in the inspection image. The geometrical features selected for classifying the defects are computationally simple to extract. Their discriminatory qualities are also high. Each of these geometrical features is discussed and justified in figure 5. 
(i)Height of defect window, $H D W$ : it is one of the noticeable discriminatory characteristics of the defects. Height of defect window of vertical missing yarn and horizontal missing yarn should be large and small, respectively. Height of defect window of color yarn should also be small. Height of defect window of hole should vary from small to medium, whereas spot's should vary from medium to large.

4.3. An Appropriate Set of Features.

(ii) Width of defect window, $W$ : it is also one of appropriate set of features are selected for classifying the defects. The features are encountered from the noticeable discriminator $D W$ characteristics of the geometrical point of view. So the features are of same type, namely geometrical feature. Geometrical features describe different discriminatory geometrical defects. Width of defect window of horizontal missing yarn and vertical missing yarn should be large and small, respectively. Width of defect

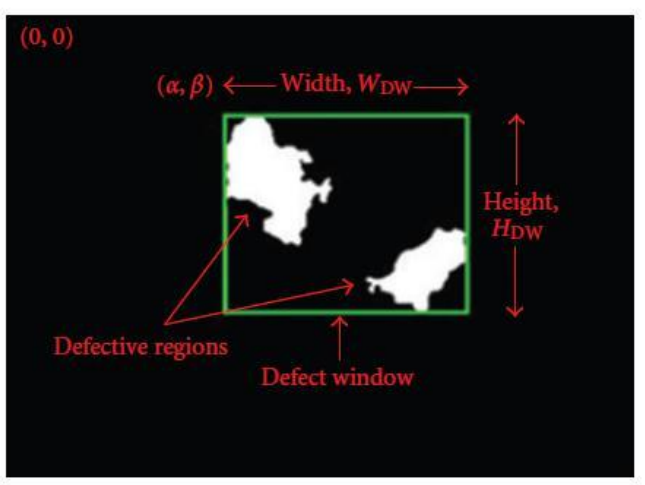

Figure 5: Description of geometric features.

Window of color yarn should also be small. Width of defect window of hole should vary from small to medium, whereas spot's should vary from medium to large.

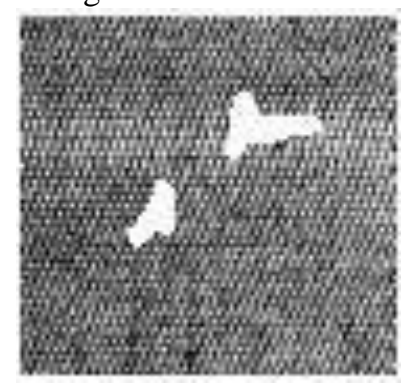

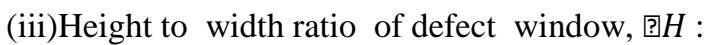

$W$ size of defect window gives a clue to a discriminatory characteristic of the defects, namely, height-to-width ratio of the defect window.

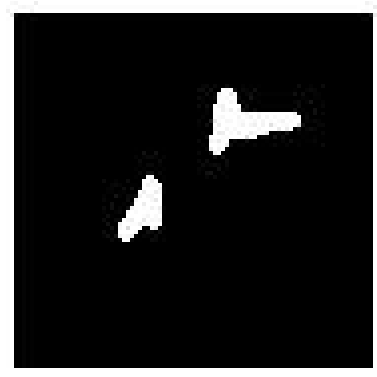

回 $W=$

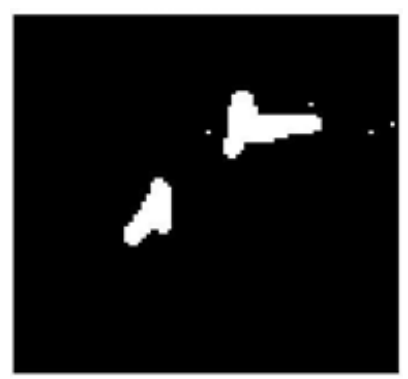




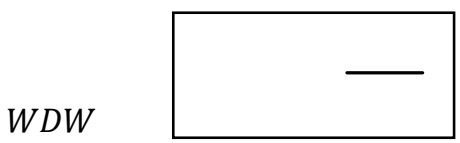

(iv) Number of defective regions, [? $D$ ? : it represents a distinguishing characteristic of spot from other defects'. The number of defective regions for spot is more than 1 in most of the cases, whereas the number of defective regions for all other defect is 1 .

\section{Result}

In this project the calculated features for the selected image are as follows:

Height of defect window, HDW=38

Width of defect window, WDW=45

Height to Width ratio of defect window, RHW $=0.8444$

Number of defective regions, NDR=289

Relative defect area $=3.5679$

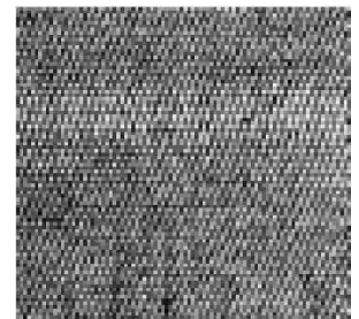

(iv) Number of defective regions, ? $D$ ? : it represents a distinguishing characteristic of spot from other defects'. the number of defective regions for spot is more than 1 in most of the cases, whereas the number of defective regions for all other defect is 1 .

\section{Result}

In this project the calculated features for the selected image are as follows:

Height of defect window, HDW $=38$

Width of defect window, WDW $=45$

Height to Width ratio of defect window, RHW $=0.8444$

Number of defective regions, NDR=289

Relative defect area $=3.5679$
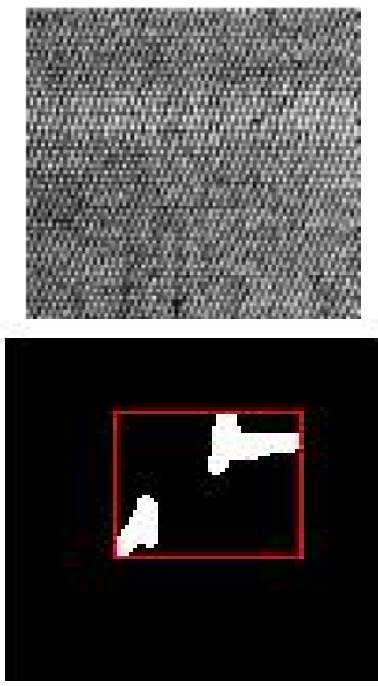

FIGURE 6. (i)input image (ii)input defected image (iii)segmented image (iv)noise removed segmented image (v) final image 


\section{References}

[1]. A. Kumar, "Neural network based detection of local textile defects," Pattern Recognition, vol. 36, no. 7, pp. 1645-1659, 2003

[2]. [D. Anderson and G. McNeill, "Artificial Neural Networks Technology," Contract Report, for Rome Laboratory, contract no. F30602-89-C-0082, August 1992.

[3]. Y. Shu and Z. Tan, "Fabric Defects Automatic Detection Using Gabor Filters," World Congress on Intelligent Control and Automation (WCICA 2004), Hangzhou, China, vol. 4, pp. 3378 - 3380, June 2004.

[4]. M. Salahudin and M. Rokonuzzaman, "Adaptive Segmentation of Knit Fabric Images for Automated Defect Detection in Semistructured Environments," Proceedings of the 8th ICCIT, pp. 255-260, 2005.

[5]. W. Jasper, J. Joines, and J. Brenzovich, "Fabric Defect Detection Using a Genetic Algorithm Tuned Wavelet Filter," Journal of the Textile Institute, vol. 96, Issue 1, pp. 43 - 54, January 2005

[6]. R. Stojanovic, P. Mitropulos, C. Koulamas, Y.A. Karayiannis, S. Koubias, and G. Papadopoulos, "Real-time Vision based System for Textile Fabric Inspection," Real-Time Imaging, vol. 7, no. 6, pp. 507-518, 2001.

[7]. "A review of automatic fabric defect detection techniques"Kolhe S.R.advances in computational research,ISSN:0975- 3273,2009 pp 18-29

[8]. R. G. Saeidi, M. Litifi, S. S. Najar, and A.G. Saeidi, "Computer vision-aided fabric inspection system for on-circular knitting machine," Textile Research Journal, vol. 75, no. 6, pp. 492-497, 2005.

[9]. F. S. Cohen, Z. Fan, and S. Attali, "Automated inspection of textile fabrics using textural models," IEEE Transactions on Pattern Analysis and MachinIntelligence, vol. 13, no. 8, pp. 803-808, 1991

[10]. A. Kumar, "Computer-vision-based fabric defect detection: a survey," IEEE Transactions on Industrial Electronics, vol. 55, no.1, pp. 348-363, 2008.

[11]. D. Phillips, Image Processing in C, R \& D Publications, Lawrence,Kan, USA, 2nd edition,2000.

[12]. C. F. J. Kuo and C. J. Lee, “A back-propagation neural networkfor recognizing fabric defects," 